\title{
Testing Basic Gradient Turbulent Transport Models for Swirl Burners Using PIV and PLIF
}

\author{
Alexey Savitskii ${ }^{1,2}$, Aleksei Lobasov ${ }^{1,2}$, Dmitriy Sharaborin ${ }^{1,2}$ and Vladimir Dulin $1,2, *$ (D) \\ 1 Kutateladze Institute of Thermophysics, 1 Lavrentyev Avenue, 630090 Novosibirsk, Russia; \\ a.savitskii@g.nsu.ru (A.S.); alexey.lobasov@gmail.com (A.L.); sharaborin.d@gmail.com (D.S.) \\ 2 Department of Physics, Novosibirsk State University, 2 Pirogov Street, 630090 Novosibirsk, Russia \\ * Correspondence: vmd@itp.nsc.ru; Tel.: +7-383-3356684
}

Citation: Savitskii, A.; Lobasov, A.; Sharaborin, D.; Dulin, V. Testing Basic Gradient Turbulent Transport Models for Swirl Burners Using PIV and PLIF. Fluids 2021, 6, 383. https://doi.org/ $10.3390 /$ fluids 6110383

Academic Editor: Martin Skote

Received: 19 August 2021

Accepted: 18 October 2021

Published: 25 October 2021

Publisher's Note: MDPI stays neutral with regard to jurisdictional claims in published maps and institutional affiliations.

Copyright: (c) 2021 by the authors. Licensee MDPI, Basel, Switzerland. This article is an open access article distributed under the terms and conditions of the Creative Commons Attribution (CC BY) license (https:// creativecommons.org/licenses/by/ $4.0 /)$.
Abstract: The present paper reports on the combined stereoscopic particle image velocimetry (PIV) and planar laser induced fluorescence (PLIF) measurements of turbulent transport for model swirl burners without combustion. Two flow types were considered, namely the mixing of a free jet with surrounding air for different swirl rates of the jet $\left(\operatorname{Re}=5 \times 10^{3}\right)$ and the mixing of a pilot jet $\left(\operatorname{Re}=2 \times 10^{4}\right)$ with a high-swirl co-flow of a generic gas turbine burner $\left(\operatorname{Re}=3 \times 10^{4}\right)$. The measured spatial distributions of the turbulent Reynolds stresses and fluxes were compared with their predictions by gradient turbulent transport models. The local values of the turbulent viscosity and turbulent diffusivity coefficients were evaluated based on Boussinesq's and gradient diffusion hypotheses. The studied flows with high swirl were characterized by a vortex core breakdown and intensive coherent flow fluctuations associated with large-scale vortex structures. Therefore, the contribution of the coherent flow fluctuations to the turbulent transport was evaluated based on proper orthogonal decomposition (POD). The turbulent viscosity and diffusion coefficients were also evaluated for the stochastic (residual) component of the velocity fluctuations. The high-swirl flows with vortex breakdown for the free jet and for the combustion chamber were characterized by intensive turbulent fluctuations, which contributed substantially to the local turbulent transport of mass and momentum. Moreover, the high-swirl flows were characterized by counter-gradient transport for one Reynolds shear stress component near the jet axis and in the outer region of the mixing layer.

Keywords: swirling flows; vortex breakdown; coherent structures; gradient diffusion hypothesis; Boussinesq's hypothesis; turbulent Schmidt number; planar laser induced fluorescence; particle image velocimetry; proper orthogonal decomposition

\section{Introduction}

Burners with flow swirl are commonly used for stabilization of flames with a compact flame zone for a wide range of fuel and oxidant flowrates. For high swirl rates, the flame stabilization is provided by a central recirculation zone where hot combustion products preheat the reactants and supply them with chemical radicals. On the other side, the dynamics of high-swirl flows is often unsteady due to the breakdown and precession of the vortex core, which produce strong velocity and pressure pulsations [1,2].

To achieve low NOx emissions, the fuel in modern combustors is burned under fuel-lean conditions after premixing with air [3]. It is important to organize steady wellpremixed combustion to avoid temperature or fuel/air ratio fluctuations to maintain high efficiency of combustion. However, lean flames are known to be sensitive to external disturbances [4-6], which can lead to thermoacoustic pulsations in the combustion chamber, reduced combustion efficiency, and flame blow-off. Therefore, the organization of steady lean premixed combustion in swirl burners is important and relies on detailed studies of flow dynamics and mixing processes. 
PIV and PLIF techniques are now widely used to measure the spatial distributions of flow velocity and concentration of fuel (by imaging the fluorescence of the fuel or a special additive) [7,8]. Measurements of these quantities can be performed simultaneously by combining the PIV and PLIF methods. Moreover, the latter provides important information about the shape of chemical reaction zones in combustors $[9,10]$. However, detailed optical measurements for the real combustion chambers are often difficult due to a number of technical reasons. Therefore, the main properties of turbulent transport and mixing mechanisms are studied for smaller burners that physically model real combustors.

Large-eddy simulation provides reliable predication of turbulent mixing and combustion for swirl burners [11,12]. However, for the purpose of optimization, prompt simulation of fuel mixing and burning in large-scale furnaces and gas turbine combustors at realistic flow rates still often relies on the use of Reynolds averaged Navier-Stokes (RANS) methods and requires experimental validation. The main option is to use a suitable turbulence closure model that allows accurate numerical calculations of the flow characteristics.

The basic closure model for the Reynolds stresses is Boussinesq's hypothesis (BH), according to which the Reynolds stresses are assumed to be proportional to the strain rate of the mean velocity, whereas the proportionality coefficient is the double turbulent (eddy) viscosity. The simplest RANS approach to model the turbulent flux of a scalar is gradient diffusion hypothesis (GDH). In this model, the fluxes are proportional to the mean concentration gradient with the turbulent diffusivity coefficient, which is assumed to be equal to the turbulent viscosity, scaled by a turbulent Schmidt number $[13,14]$.

To account for turbulence anisotropy during the transport of passive scalar, Daly and Harlow [15] suggested the use of turbulent diffusivity tensor instead of single scalar value. In this case, the components of turbulent diffusivity tensor are assumed to be proportional to the Reynolds stresses. Moreover, it is known that the Boussinesq's approximation assumes the locally isotropic properties of turbulence and may fail to provide accurate prediction for flows with swirl [16]. To account for the anisotropy of turbulent fluctuations and overcome the closure restriction, Reynolds stresses transport equations can be solved [17]. However, this method is computationally more expensive and involves more empirical parameters.

Paglianti and Montante [18] studied mixing in a continuous flow stirred tank using the PIV and PLIF methods simultaneously. The authors evaluated the turbulent viscosity and diffusivity coefficients with the corresponding turbulent Schmidt number based on gradient diffusion and Boussinesq's hypotheses, respectively. They concluded that these closure models did not fit the measured data. Nevertheless, the obtained values of the turbulent Schmidt number were in a range recommended for numerical simulations.

Hitimana et al. [19] investigated turbulent mixing in a multi-inlet vortex reactor. Combined PLIF and PIV measurements were performed to evaluate the turbulent viscosity, turbulent diffusivity, and turbulent Schmidt number. They showed that the Schmidt number value for the experimental data was not a constant. They also outlined that the highest turbulent Schmidt number variations corresponded to the regions with the highest magnitude of the concentration gradient.

The focus of the present paper is on the capability of the basic gradient turbulent transport models (BH and GDH) to predict turbulent shear stress and turbulent fluxes during mixing for a free jet flow configuration and for a pilot jet of a generic gas turbine swirler in a combustion chamber. Special emphasis was placed on unsteady high-swirl flows with vortex breakdown and intensive coherent flow fluctuations. In this case, the unsteady fluctuations were extracted using POD, and the gradient transport models were also tested for the stochastic (residual) component of the velocity and concentration fluctuations.

\section{Experimental Setup}

\subsection{Test Facilities}

An axisymmetric contraction nozzle was used to organize the free jet flows. Flow swirl was induced by a changeable vane swirler, which was mounted inside the nozzle 
(additional details are provided in [20]). The values of the swirl rate $S$ defined by (1) were $0.41,0.7$, and 1.0. In Equation (1), $d_{1}=7 \mathrm{~mm}$ is the center body diameter, $d_{2}=27 \mathrm{~mm}$ is the external diameter of the swirler, and $\psi$ is the inclination angle of the swirler's vanes relative to the axis. The swirl rate was changed using swirlers with different $\psi$.

$$
S=\frac{2}{3}\left(\frac{1-\left(d_{1} / d_{2}\right)^{3}}{1-\left(d_{1} / d_{2}\right)^{2}}\right) \tan (\psi)
$$

The Reynolds number of the free jets, which was calculated using the bulk velocity $U_{0}$ of the flow, the nozzle exit diameter $d=15 \mathrm{~mm}$, and the kinematic viscosity of the air $v$, was $\operatorname{Re}=U_{0} d / v=5 \times 10^{3}$. Mass flow meters (Bronkhorst) were used to control the flowrate of the air. Acetone vapor ( $3 \%$ by volume) was added into the flow by bubbling a part of the air flow through a heated reservoir with liquid acetone. The air flow was also passed through a mechanical mixer to seed it with $\mathrm{TiO}_{2}$ particles with a mean diameter of $0.5 \mu \mathrm{m}$. A fog machine was used to seed the surrounding air.

The experiments were also carried out for a model gas turbine swirl combustor under atmospheric conditions. The combustor included a plenum chamber, a model gas turbine swirler based on a design by Turbomeca [21], a combustor with optical windows made of fused silica, and an exhaust contraction nozzle. The gas fuel were supplied in two ways: as a pilot jet through a centerbody of the swirler or through holes between the swirler's vanes. Details on the geometry can be found in [22]. The main air flow was seeded by $\mathrm{TiO}_{2}$ particles. To study the flow and mixing of the central jet, it was also seeded by $\mathrm{TiO}_{2}$ particles and by acetone vapor. Air, methane, or neon (for the same mass flowrate) were used for the central jet to investigate the effect of the gas density.

\subsection{PIV/PLIF Equipment}

Simultaneous measurements of the instantaneous acetone concentration and flow velocity were carried out by a combined system of the PLIF and stereo-PIV measurement methods. The scheme of the experimental setup is shown in Figure 1. The stereo-PIV system included a pair of CCD cameras (ImperX Bobcat IGV-B2020, 4 Mpix), which were equipped with narrow-band optical filters for $532 \pm 5 \mathrm{~nm}$. A double-head pulsed Nd:YAG laser (Quantel EverGreen 200) was used to illuminate the tracer particles. The PLIF system included a tunable pulsed dye laser (Sirah Precision Scan) with a wavelength of approximately $283 \mathrm{~nm}$, pumped by a pulsed Nd:YAG laser (QuantaRay). A sCMOS camera (LaVision, 5 Mpix, 16 bit) with a UV-sensitive image intensifier (LaVison IRO with S20 photocathode) was used to capture the acetone fluorescence images. The PLIF camera and intensifier were equipped with UV lens and optical band pass filters for 415-455 nm.

To correct for the PLIF laser sheet nonunimormity and energy pulse instability, part of the laser sheet was reflected into a cuvette with a Rhomadine 6G solution. Another CCD camera was used to capture the fluorescence inside the cuvette. The laser pulse energy was varied to validate a linear regime of the fluorescence. Synchronization of the PIV/PLIF cameras and lasers was provided by a TTL signal generator. The pulse of the PLIF laser was between the pair of PIV laser pulses. The PLIF camera exposition was 200 ns. The PIV and PLIF cameras were spatially calibrated by placing a special calibration plate with markers. Mapping function that modeled the projections of the measurement plane to the sensors of the PIV and PLIF cameras was obtained during spatial calibration. 


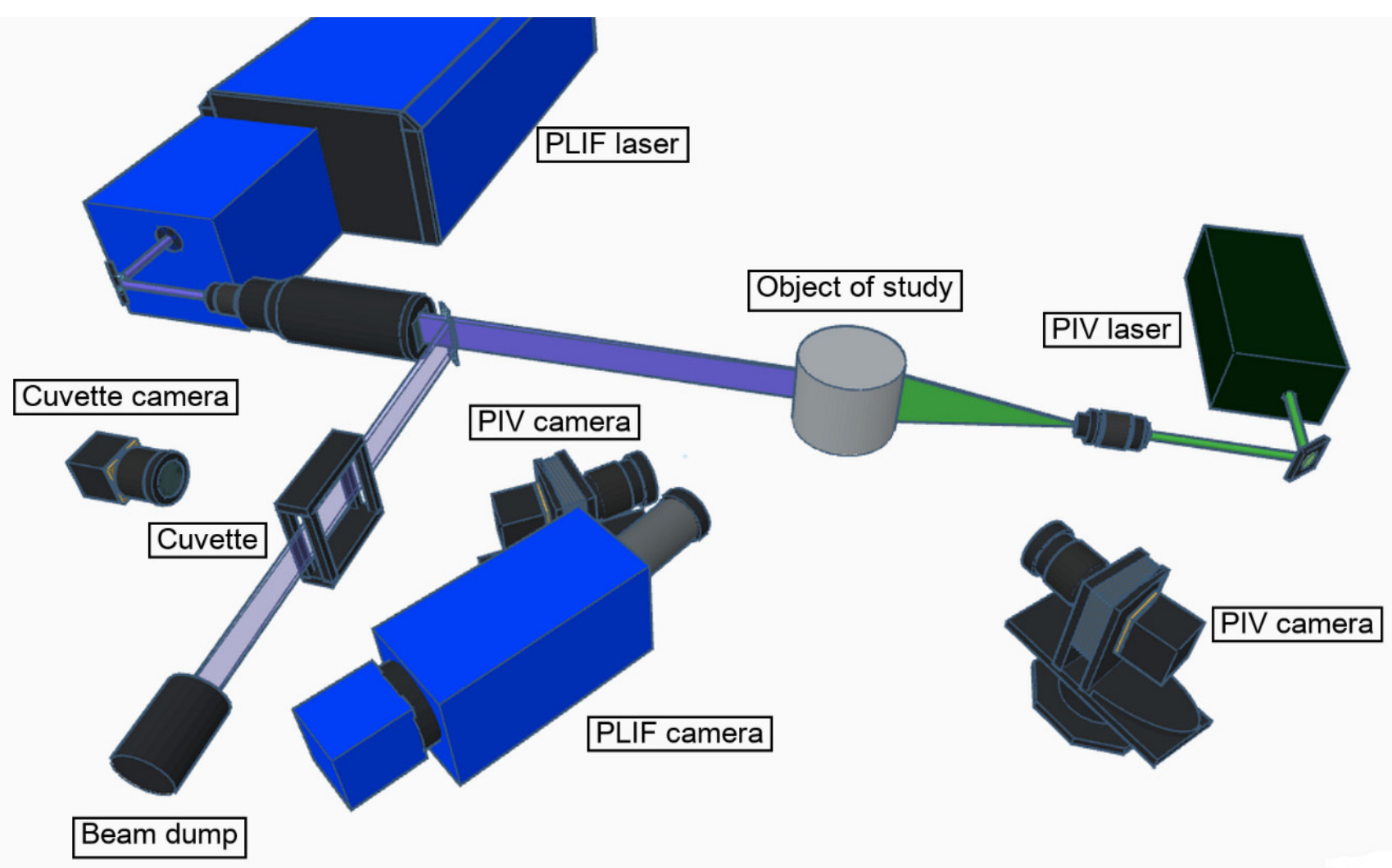

Figure 1. The scheme of the experimental setup.

\subsection{Data Processing}

PIV data were acquired and processed using an "Actual Flow" software developed in the Institute of Thermophysics. An adaptive cross-correlation based algorithm with continuous shift and deformation of interrogation areas [23] was exploited to evaluate the local velocity field of the tracers. The size of the final integration areas was $32 \times 32$ pixels. The spatial overlap rate between the areas was $50 \%$. A pair of mapping functions captured during the spatial calibration prior to the experiment and a pair of two component 2D velocity fields captured during the measurements were used to perform the stereo reconstruction of a three-component 2D velocity field [24]. Back-projection and spatial averaging were performed for the PLIF data to fit the PIV data grid.

The following algorithm was applied to process PLIF data. First, the background signal, which included a dark current, background, and reflections, was removed. The background signal was captured when the laser illuminated the measurement plane without the flow. Next, the nonuniform sensitivity of the detector was considered by imaging a defocused white paper sheet. The laser sheet nonuniformity was corrected based on the laser sheet imaging inside the cuvette. Finally, Beer-Lambert law was used to correct for the absorption attenuation of PLIF laser sheet by the acetone molecules for each image.

For each flow case of the free jet and for the methane pilot jet mixing in the gas turbine combustor, a set of 1500 velocity and concentration snapshots was measured. For the cases of air and neon pilot jets, only 500 snapshots were used. The fluctuating velocity data sets $\mathbf{U}=\left[\mathbf{u}^{\prime}\left(\mathbf{x}, t_{1}\right) \ldots \mathbf{u}^{\prime}\left(\mathbf{x}, t_{N}\right)\right]$ were processed by a snapshot proper orthogonal decomposition (POD, [25]), which is based on the singular value decomposition (SVD) of the data matrix $\mathbf{U}[26]$.

$$
\mathbf{U}=\mathbf{W} \Sigma \mathbf{V}, \text { or } \mathbf{u}^{\prime}\left(\mathbf{x}, t_{k}\right)=\sum_{q=1}^{N} \alpha_{q}\left(t_{k}\right) \sigma_{q} \boldsymbol{\varphi}_{q}(\mathbf{x}),
$$




$$
\text { where } \mathbf{W} \mathbf{W}^{T}=\mathbf{I}_{M}, \mathbf{V} \mathbf{V}^{T}=\mathbf{I}_{N} \text {, or } \sum_{k=1}^{M} \boldsymbol{\varphi}_{i}\left(\mathbf{x}_{k}\right) \boldsymbol{\varphi}_{j}\left(\mathbf{x}_{k}\right)=\delta_{i j}, \sum_{k=1}^{N} \alpha_{i}\left(t_{k}\right) \alpha_{j}\left(t_{k}\right)=\delta_{i j}
$$

Here, $\delta_{i j}$ is the Kronecker symbol, $\mathbf{W}=\left[\boldsymbol{\phi}_{1}(\mathbf{x}) \ldots \boldsymbol{\phi}_{N}(\mathbf{x})\right]$ is the matrix of left-singular vectors (containing the POD modes), $\mathbf{V}$ is matrix of right-singular vectors $\left(\mathbf{V}^{T}\right.$ corresponds to the normalized temporal coefficients $\alpha_{q}$ ), and $\Sigma=\operatorname{diag}\left[\sigma_{1} \ldots \sigma_{N}\right]$ is the matrix of singular values. When the flow dynamics is dominated by coherent fluctuations, the first two POD modes can be used to obtain a phase-averaged velocity distribution $[27,28]$, where $\varphi$ is the phase.

$$
\hat{\mathbf{u}}(\mathbf{x}, \phi)=\left(\sigma_{1} / \sqrt{N}\right) \sin (\phi) \boldsymbol{\varphi}_{1}(\mathbf{x})+\left(\sigma_{2} / \sqrt{N}\right) \cos (\phi) \boldsymbol{\varphi}_{2}(\mathbf{x})
$$

Therefore, the POD can be used to decompose the velocity fluctuations into the coherent pulsations and residual (stochastic) part [29].

$$
\mathbf{u}^{\prime}=\hat{\mathbf{u}}+\mathbf{u}^{\prime \prime}
$$

Because the POD is based on the SVD, which provides the orthonormal spatial modes and orthonormal temporal coefficients, it ensures zero correlation between the stochastic and coherent fluctuations.

$$
\left\langle u_{i} u_{j}\right\rangle=\left\langle\hat{u}_{i} \hat{u}_{j}\right\rangle+\left\langle\hat{u}_{i} u_{j}^{\prime \prime}\right\rangle+\left\langle u_{i}^{\prime \prime} \hat{u}_{j}\right\rangle+\left\langle u_{i}^{\prime \prime} u_{j}^{\prime \prime}\right\rangle=\left\langle\hat{u}_{i} \hat{u}_{j}\right\rangle+\left\langle u_{i}^{\prime \prime} u_{j}^{\prime \prime}\right\rangle
$$

Afterwards, the concentration fluctuation fields $\mathbf{C}=\left[c^{\prime}\left(\mathbf{x}, t_{1}\right) \ldots c^{\prime}\left(\mathbf{x}, t_{N}\right)\right]$ can be phase-averaged to obtain the coherent pulsations of the concentration $[20,30]$.

$$
\hat{c}_{q}(\mathbf{x})=(1 / \sqrt{N}) \sum_{k=1}^{N} a_{q k} c^{\prime}\left(\mathbf{x}, t_{k}\right)
$$

Similar conditions apply for the correlations between the coherent and stochastic fluctuations of the concentration.

$$
\left\langle u_{i} c\right\rangle=\left\langle\hat{u}_{i} \hat{c}\right\rangle+\left\langle\hat{u}_{j} c^{\prime \prime}\right\rangle+\left\langle u_{i}^{\prime \prime} \hat{c}\right\rangle+\left\langle u_{i}^{\prime \prime} c^{\prime \prime}\right\rangle=\left\langle\hat{u}_{i} \hat{c}\right\rangle+\left\langle u_{i}^{\prime \prime} c^{\prime \prime}\right\rangle
$$

\section{Results}

\subsection{Flow Structure and Coherent Fluctuations}

Figure 2 shows the mean concentration and velocity fields for the free jets with different swirl rates. The studied flows are described using the cylindrical coordinate system with radial, axial, and tangential coordinates $(r, y$, and $\theta$, respectively). The origin is defined at the center of the nozzle outlet. Therefore, $\left(U_{r}, U_{y}, U_{\theta}\right)$ and $\left(u_{r}, u_{y}, u_{\theta}\right)$ are the time-averaged and instantaneous velocity vectors with the radial, axial, and tangential components, respectively. 2D plots show data for the central plane $(x-y)$ of the flow, where $x$ is the transverse coordinate. For better visualization of the velocity fields, each 6th and each 4th vector in the $y$ direction is shown for the free swirling jets and the swirling jets in the combustion chamber, respectively.

The jet with $S=0.41$ is referred to as a low-swirl jet because the swirl was sufficient enough to produce the central wake where the mean velocity remained positive. It indicated that there was central recirculation zone. The jets with $S=0.71$ and 1.0 are referred to as high-swirl jets because they both had central recirculation zones. In general, an increase in swirl rate results in an increase in the jet due to faster mixing with the surrounding air. Figure 1 also shows examples of the instantaneous velocity and concentration snapshots. It demonstrates that large-scale vortex structures developed in the mixing layers and provided mass transfer between the jet and surrounding air. 

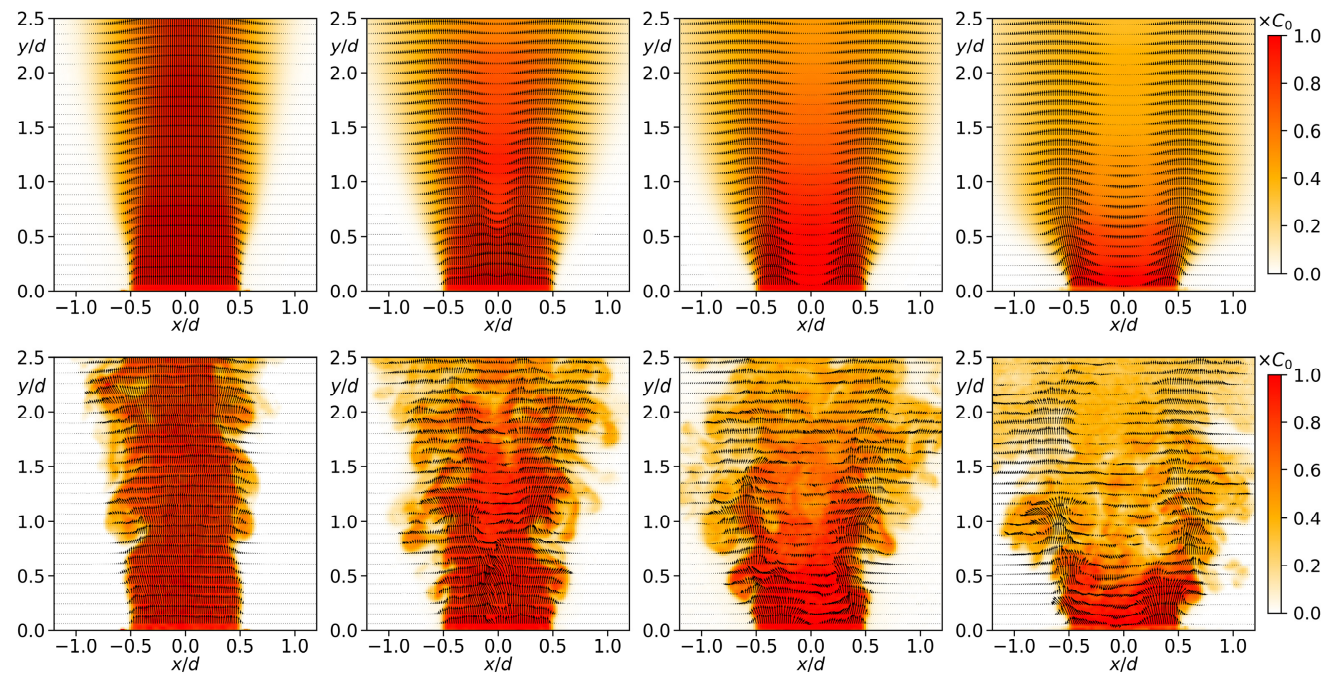

(a)

(b)

(c)

(d)

Figure 2. Mean and instantaneous concentration and velocity fields for jet flows: (a) $S=0$, (b) $S=0.41$, (c) $S=0.7$, and (d) $S=1.0$.

Figure 3 shows the time-averaged and instantaneous distributions for the swirling flow in the combustion chamber with the central jet of air, methane, and neon. The flow swirl was high and resulted in the presence of a central recirculation zone, which was penetrated by the central jet. For the methane, the jet velocity was higher and it penetrated further inside the recirculation zone. Moreover, the methane jet mixed faster in comparison to neon and air. For the free jets and for the flow of the model gas turbine swirler, the fuel mixing was related to the unsteady transport by large-scale vortical structures. POD was used to extract the coherent velocity fluctuations produced by them.
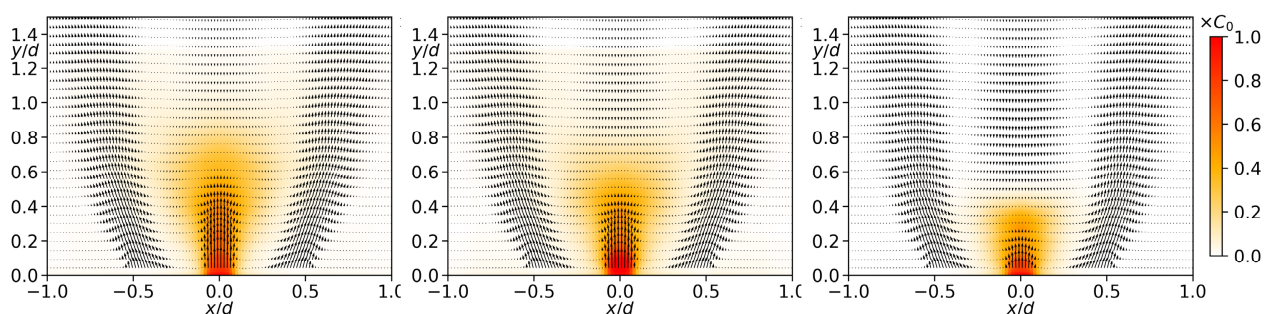

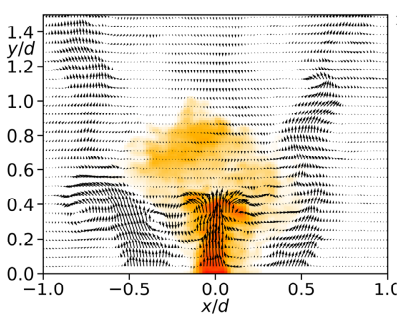

(a)

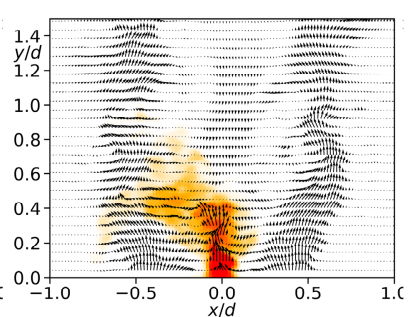

(b)

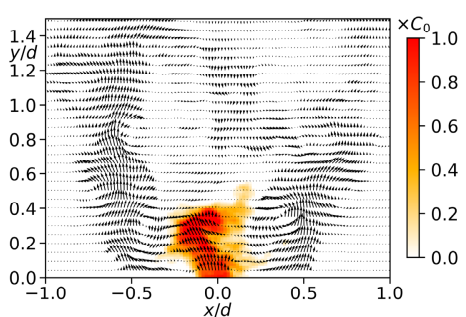

(c)

Figure 3. Mean and instantaneous concentration and velocity fields for gas turbine combustor with pilot jet of (a) methane, (b) neon, and (c) air.

Figure 4 shows the POD spectra of velocity fluctuations for the free jets with various swirl rate and for the flow of the model gas turbine combustor. The insets show the distributions of the temporal coefficients of the first two POD modes for the free jet with swirl rate of 1.0 (a) and for the gas turbine combustor with methane pilot jet (b). For the cases of the free swirling jets, the coherent velocity fluctuations for the swirl rates of 
0.7 and 1.0 were related to the first two POD modes, which corresponded to the phase shift of $\pi / 2$. The kinetic energy of these two modes was considerably greater than that of each remaining mode, namely it exceeded $9 \%$ and $16 \%$ of the spatially averaged turbulent kinetic energy for the cases $S=0.7$ and 1.0, respectively. For the gas turbine swirler, the POD for all cases revealed the presence of coherent fluctuations, which were related to the first two POD modes.

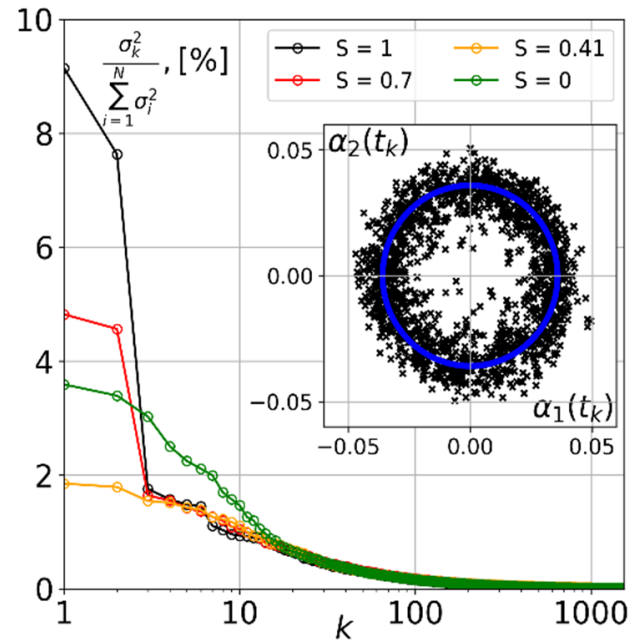

(a)

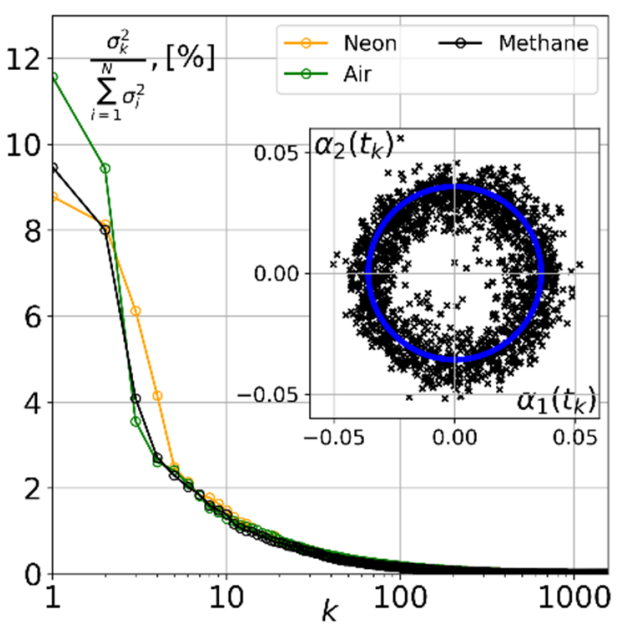

(b)

Figure 4. POD spectra and temporal coefficients for the first two POD modes (inset): (a) turbulent swirling jets and (b) model gas turbine combustor.

Figure 5 shows the anisotropy invariant maps for the coherent and stochastic components of the velocity fluctuations of the nonswirling and low-swirl jets without vortex breakdown. The data was taken along the mixing layers of the jets (for $r / d \approx 0.55$ ). Because the flows were not characterized by intensive coherent velocity fluctuations, the scatter plots for both fluctuation components were quite similar. For the nonswirling jet, the data corresponded to the axisymmetric expansion during the flow issue from the nozzle and formation of ring-like vortex structures in the mixing layer. For the low-swirl jet, the scatter plot was away from the axisymmetric expansion due to the development of azimuthal modes (see 3D measurements in [31]).

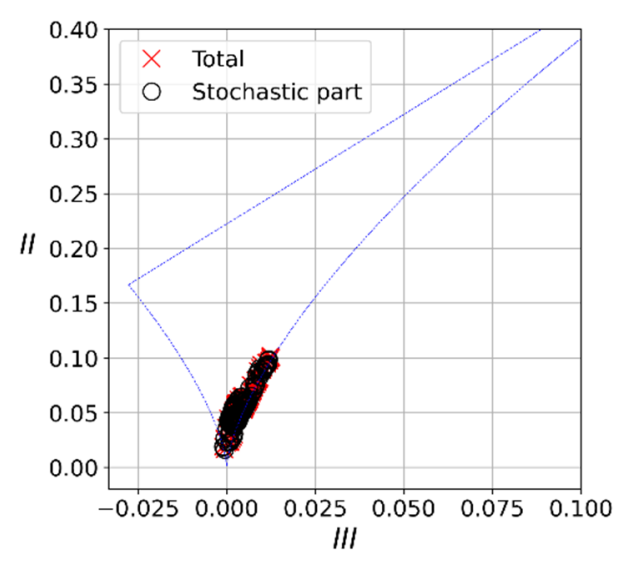

(a)

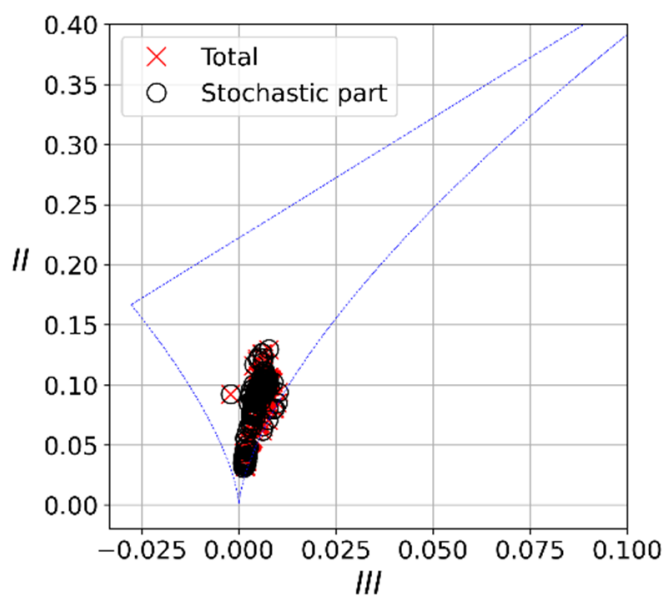

(b)

Figure 5. Turbulence anisotropy invariant map for (a) nonswirling and (b) low-swirl jets. 
It is important to characterize the spatial resolution of the PIV system used (the final interrogation area size was $\approx 0.96 \mathrm{~mm}$ ) relative to the scales of turbulent fluctuations. The integral scale for the free jets was of the order of the nozzle diameter, i.e., $\sim 15 \mathrm{~mm}$. The fine scale could be evaluated based on $\operatorname{Re}^{3 / 4}$ scaling or from the $\left(v^{3} / \varepsilon\right)^{1 / 4}$ scaling. Because direct measurements were not possible in the present experiment, the magnitude of the turbulent kinetic energy dissipation rate $\varepsilon$ was roughly evaluated from the maximal shear production of the turbulent kinetic energy:

$$
P=\frac{\partial U_{r}}{\partial y}\left\langle u_{r}^{\prime} u_{y}^{\prime}\right\rangle
$$

The finest scales were evaluated to be in the range of 30 to $75 \mu \mathrm{m}$ for the studied free swirling jets. It is important to note that despite the spatial resolution not being sufficient to resolve the velocity and concentration gradients, it allowed the majority of the turbulent kinetic energy of large-scale velocity and concentration fluctuations to be captured. As was shown in [20], the resolution error for the second-order statistical moments should be below $15 \%$.

The turbulence anisotropy invariant maps for the high-swirl flows with vortex breakdown of the free jet and gas turbine swirler are shown in Figure 6. The data was taken across the flows at $(y / d=0.2)$. In these cases, the flow fluctuations were strongly affected by the coherent mode and the stochastic fluctuations tended to scatter closer to the isotropic limit. The fine scale for the turbulent fluctuations in the gas turbine swirler flow was evaluated to be in the range of $15-50 \mu \mathrm{m}$, whereas the spatial resolution of the PIV system was $1.06 \mathrm{~mm}$. The spatial resolution effect on the accuracy of the second-order moments was also evaluated in [22] and reported to be below $10 \%$.

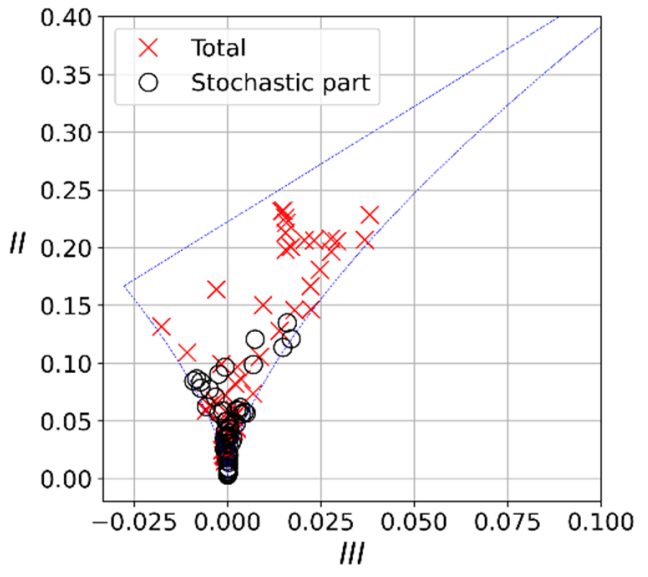

(a)

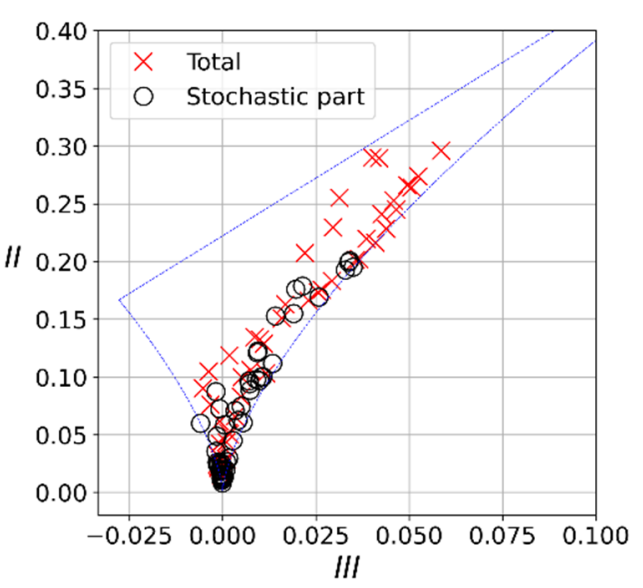

(b)

Figure 6. Turbulence anisotropy invariant map for (a) high-swirl jet $(\operatorname{Re}=5000)$ and $(\mathbf{b})$ gas turbine combustor $(\operatorname{Re}=30,000)$.

\subsection{Nonswirling Jet}

Figure 7 shows the Reynolds shear stress $\left\langle u_{r}^{\prime} u_{y}^{\prime}\right\rangle$ and Reynolds flux $\left\langle u_{r}^{\prime} c^{\prime}\right\rangle$ measured for the free jet without external swirl. The coordinates in the equations correspond to the cylindrical system, where the radial coordinate $r=x$ for $x>0$. The distributions demonstrate that the turbulent mass and momentum transport took place in the circular mixing layer of the jet. Based on these distributions, it is possible to evaluate the components of turbulent viscosity tensor (10) and turbulent diffusivity coefficient according to Equations (11)-(14).

$$
\left\langle u_{i}^{\prime} u_{j}^{\prime}\right\rangle-\frac{1}{3}\left\langle u_{k}^{\prime} u_{k}^{\prime}\right\rangle \delta_{i j}=-v_{t}\left(\frac{\partial U_{i}}{\partial x_{j}}+\frac{\partial U_{j}}{\partial x_{i}}\right)
$$




$$
\begin{gathered}
\left\langle u_{r}^{\prime} u_{y}^{\prime}\right\rangle=-v_{t, 12}\left(\frac{\partial U_{y}}{\partial r}+\frac{\partial U_{r}}{\partial y}\right) \\
\left\langle u_{r}^{\prime} u_{\theta}^{\prime}\right\rangle=-v_{t, 23}\left(r \frac{\partial\left(U_{\theta} / r\right)}{\partial r}\right) \\
\left\langle u_{y}^{\prime} u_{\theta}^{\prime}\right\rangle=-v_{t, 13} \frac{\partial U_{\theta}}{\partial y} \\
\left\langle u_{r}^{\prime} c^{\prime}\right\rangle=-D_{t, r} \frac{\partial C}{\partial r}
\end{gathered}
$$

where $U$ is the mean velocity, and $C$ is the mean concentration.

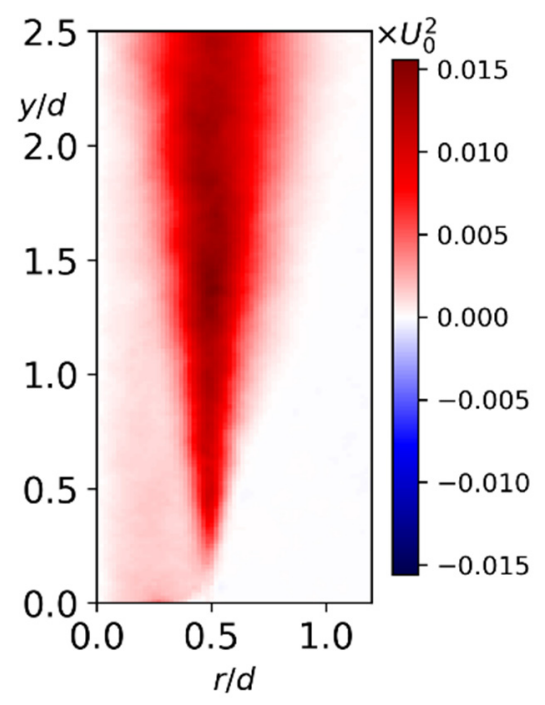

(a)

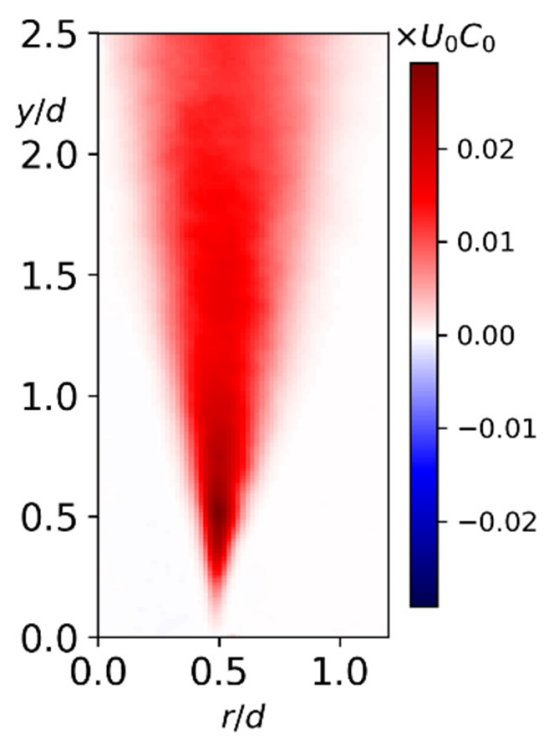

(b)

Figure 7. (a) Axial-radial Reynolds shear stress and (b) radial turbulent flux for nonswirling jet.

The evaluated coefficients are shown in Figure 8 for the radial cross-sections at different distances from the nozzle exit. The $\mathrm{BH}$ provides an accurate approximation with almost fixed value of the turbulent viscosity coefficient for the interval $0.7>r / d>0.3$, whereas the values of the turbulent diffusivity vary in the range of 0.0025 to 0.012 of $U_{0} d$ for the GDH. Consequently, the values of the turbulent Schmidt number, evaluated from (15) and shown in Figure 8c, represent the scatter from 0.2 in the center of the mixing layer up to 0.8 in the outer region of the mixing layer.

$$
S c_{t}=\frac{v_{t, 12}}{D_{t, r}}
$$

For the nonswirling jet, the other turbulent viscosity coefficients were negligible because there was no induced flow rotation. To demonstrate the swirl rate effect on the turbulent Schmidt number, the same equation was used for the flows with swirl and for the central pilot jet in the gas turbine combustor. However, it should be noted that other viscosity or diffusivity components can be used (e.g., for the rotating flows in steering mixers $[18,19])$. 


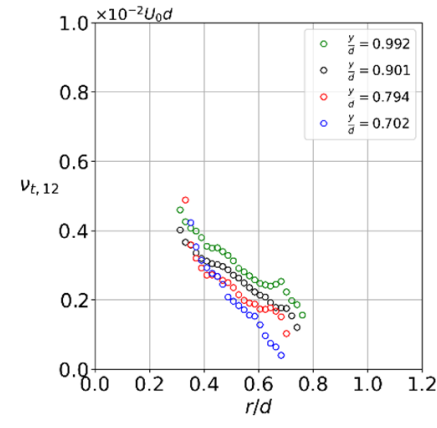

(a)

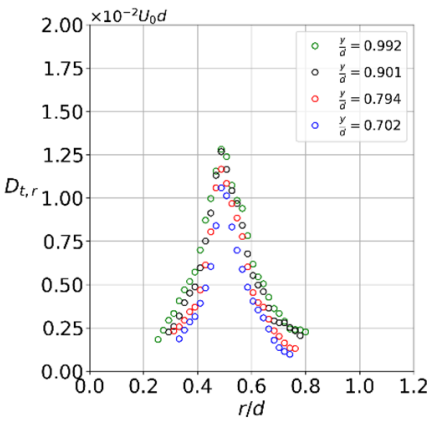

(b)

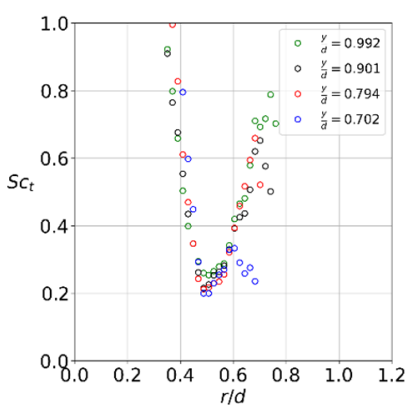

(c)

Figure 8. (a) Turbulent viscosity, (b) turbulent diffusivity, and (c) turbulent Schmidt number for nonswirling jet.

\subsection{Low-Swirl Jet}

The distributions of the Reynolds shear stresses and the radial Reynolds flux for the low-swirl jet are shown in Figure 9. The presence of the inner shear layer around the central wake resulted in a region of negative $\left\langle u_{r}^{\prime} u_{y}^{\prime}\right\rangle$ values around the jet axis. Because the flow was characterized by a swirl, the radial transfer of the angular momentum, corresponding to the positive region of $\left\langle u_{r}^{\prime} u_{\theta}^{\prime}\right\rangle$, was in the outer shear layer, where the jet entrained the surrounding air. The radial turbulent mass flux $\left\langle u_{r}^{\prime} c^{\prime}\right\rangle$ was also maximal in the outer mixing layer.

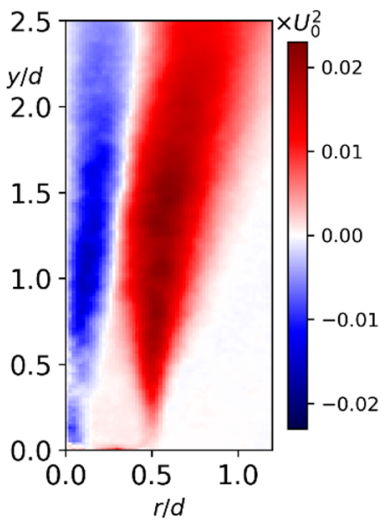

(a)

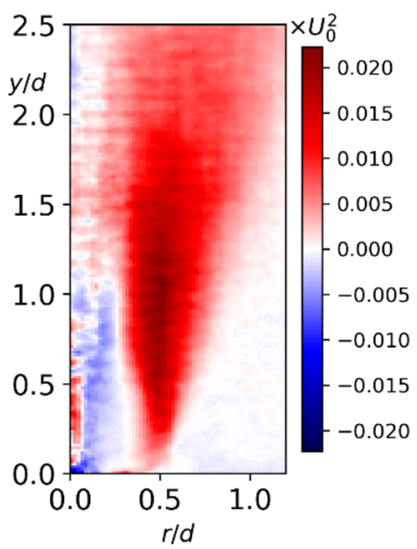

(b)

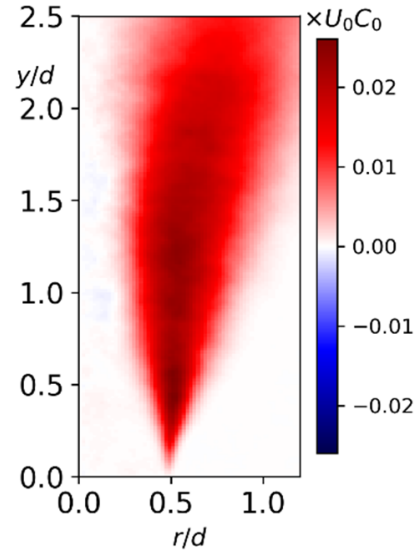

(c)

Figure 9. The distributions of Reynolds shear stresses and turbulent flux (a) $\left\langle u_{r}^{\prime} u_{y}^{\prime}\right\rangle,(\mathbf{b})\left\langle u_{r}^{\prime} u_{\theta}^{\prime}\right\rangle$, and (c) $\left\langle u_{r}^{\prime} c^{\prime}\right\rangle$ for low-swirl jet.

These distributions were used to evaluate the turbulent viscosity and diffusivity coefficients, as shown in Figure 10. For the turbulent viscosity, the proportionality between the different components of the turbulent shear stress and mean shear stress were evaluated. The coefficients had a similar shape for the outer shear layer, reaching a maximum at $r / d \approx 0.55$. The radial transport of the axial momentum $\left\langle u_{r}^{\prime} u_{y}^{\prime}\right\rangle$ was also sufficient in the inner shear layer, where the $\mathrm{BH}$ predicted the turbulent viscosity of about $0.0025 U_{0} d$. The spatial distribution of the turbulent diffusivity was similar to that in the nonswirling free jet. The evaluated turbulent Schmidt number demonstrated more uniform distribution with the value of 0.6 in the mixing layer. 


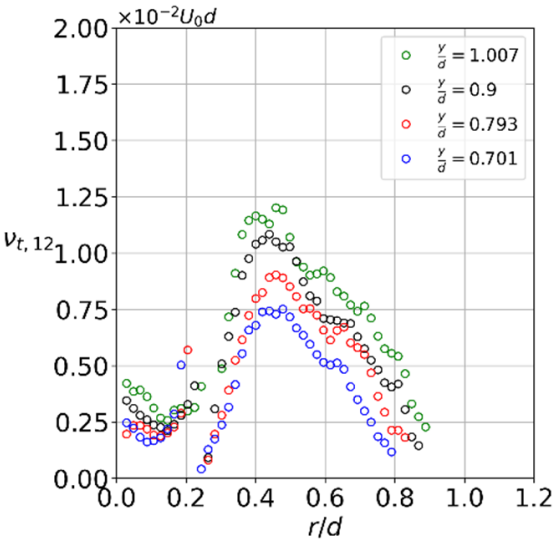

(a)

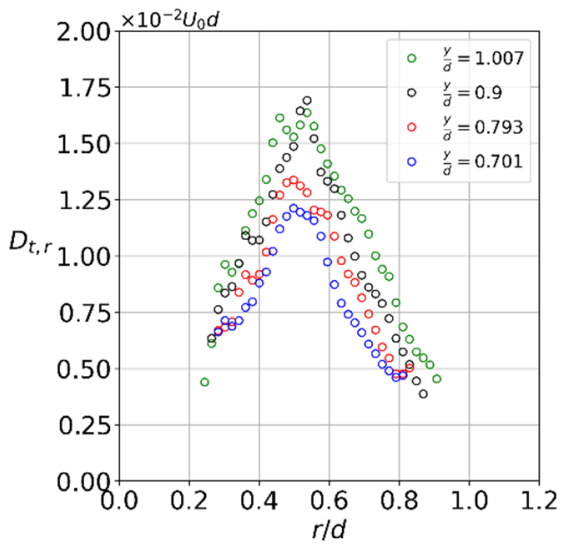

(c)

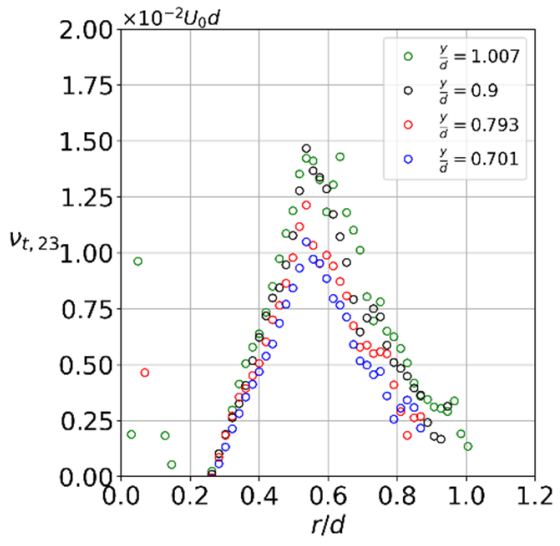

(b)

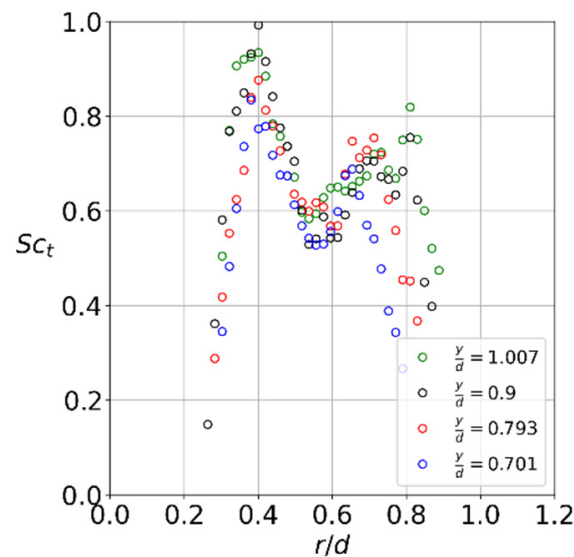

(d)

Figure 10. (a,b) Turbulent viscosity coefficients, (c) turbulent diffusion coefficient, and (d) turbulent Schmidt number for low-swirl jet.

\subsection{High-Swirl Jet}

The Reynolds shear stresses for the high-swirl jet $(S=1.0)$ with vortex breakdown are shown in Figure 11. The figure also includes these quantities for stochastic turbulent fluctuations when the coherent pulsations were removed using the first two POD modes. The coherent fluctuations contributed mainly near the nozzle exit, where they resulted in negative regions of the radial fluxes of the axial momentum $\left\langle u_{r}^{\prime} u_{y}^{\prime}\right\rangle$ and angular momentum $\left\langle u_{r}^{\prime} u_{\theta}^{\prime}\right\rangle$.

Coherent turbulent fluctuations also strongly contributed to the turbulent transport of the mass in the mixing layer. Figure 12 compares the radial flux for the total $\left\langle u_{r}^{\prime} c^{\prime}\right\rangle$ and stochastic $\left\langle u_{r}^{\prime \prime} c^{\prime \prime}\right\rangle$ components of the fluctuations. Locally, the contribution exceeded $65 \%$.

Figure 13 compares the evaluated turbulent viscosity components for different Reynolds shear stresses for total and stochastic velocity fluctuations. The $\mathrm{BH}$ was related to negative viscosity for both components $\left\langle u_{y}^{\prime} u_{\theta}^{\prime}\right\rangle$ and $\left\langle u_{y}^{\prime \prime} u_{\theta}^{\prime \prime}\right\rangle$ in the outer shear layer, where the jet entrained the surrounding air. Therefore, the counter-gradient transport took place here. A similar behavior was observed near the jet axis. For the radial flux of the angular momentum, the removal of coherent fluctuations provided a more uniform distribution of the turbulent viscosity coefficient, which was scattered around $0.01 U_{0} d$ for the jet core and in the outer shear layer. For the radial flux of the axial momentum, the $\mathrm{BH}$ provided the values of turbulent viscosity in the range of 0.005 to 0.013 of $U_{0} d$. 


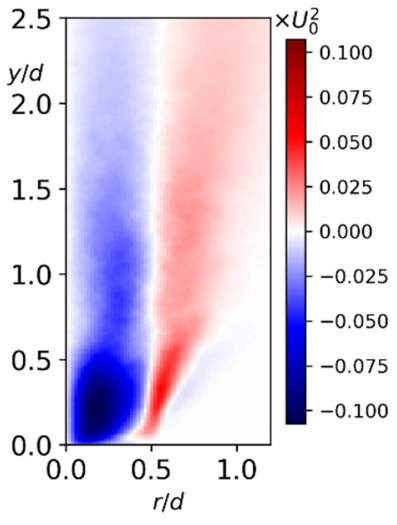

(a)

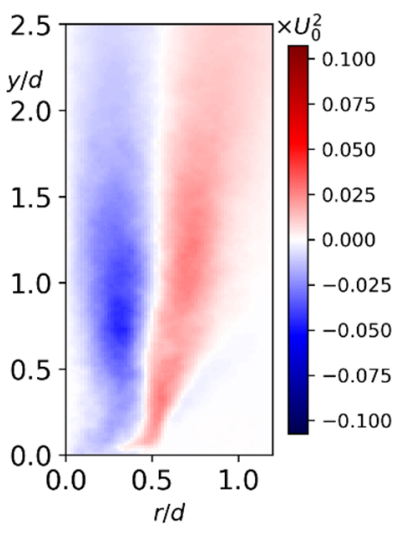

(d)

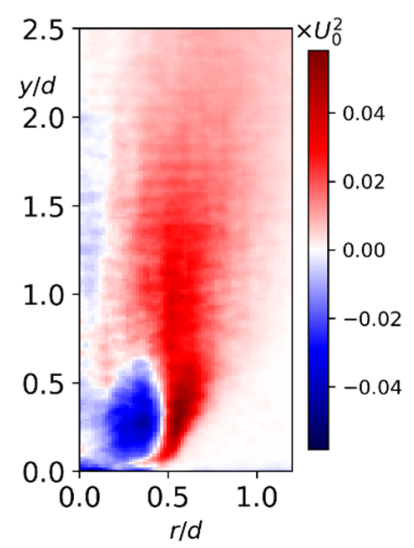

(b)

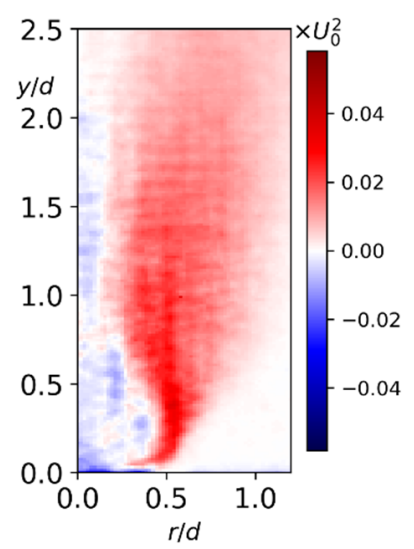

(e)

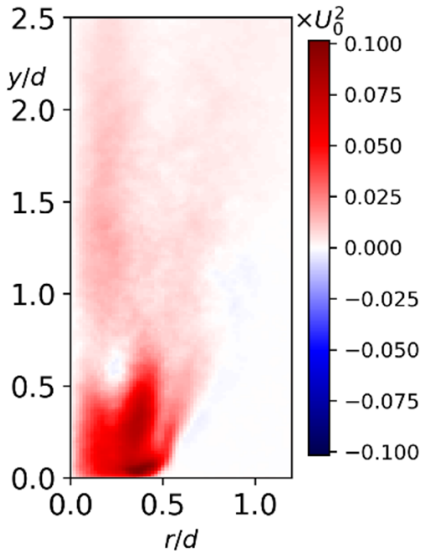

(c)

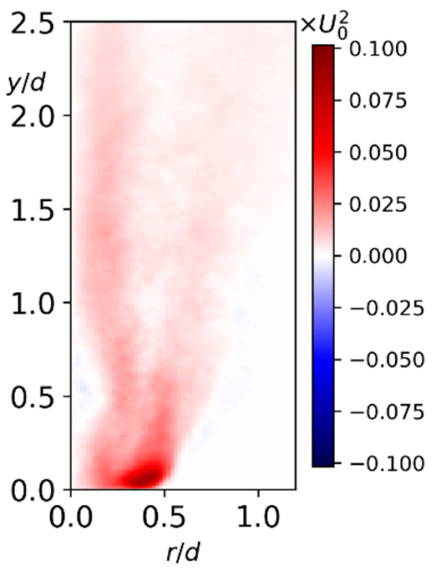

(f)

Figure 11. Distributions of Reynolds shear stresses (a) $\left\langle u_{r}^{\prime} u_{y}^{\prime}\right\rangle$, (b) $\left\langle u_{r}^{\prime} u_{\theta}^{\prime}\right\rangle$, (c) $\left\langle u_{y}^{\prime} u_{\theta}^{\prime}\right\rangle$, and the corresponding stochastic parts (d) $\left\langle u_{r}^{\prime \prime} u_{y}^{\prime \prime}\right\rangle$, (e) $\left\langle u_{r}^{\prime \prime} u_{\theta}^{\prime \prime}\right\rangle$, and (f) $\left\langle u_{y}^{\prime \prime} u_{\theta}^{\prime \prime}\right\rangle$ for high-swirl jet.

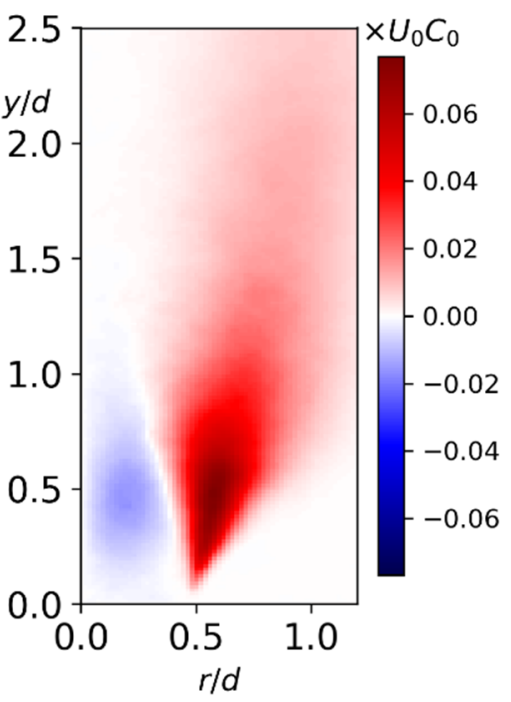

(a)

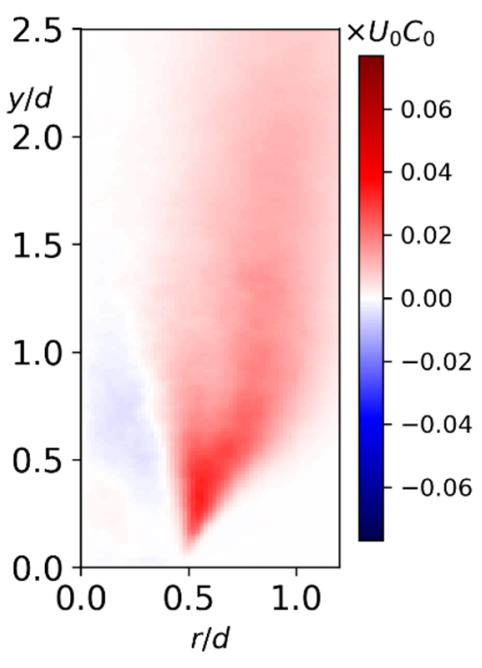

(b)

Figure 12. Radial turbulent flux for (a) total and (b) stochastic turbulent fluctuations for high-swirl jet. 


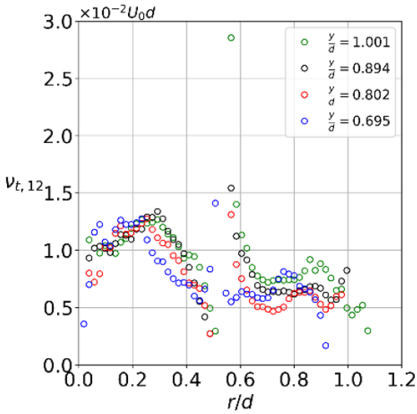

(a)

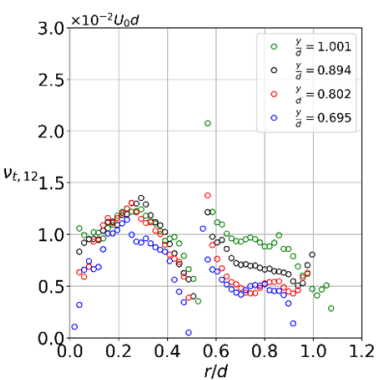

(d)

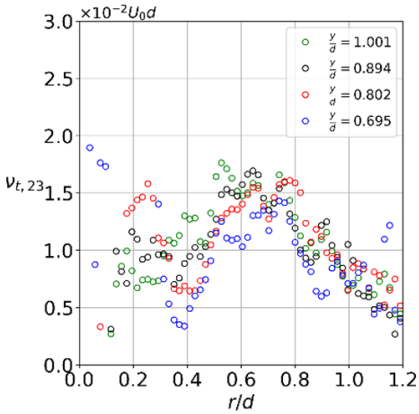

(b)

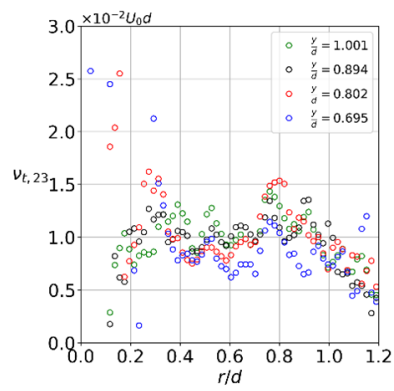

(e)

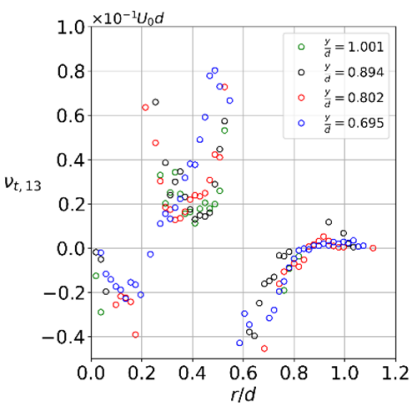

(c)

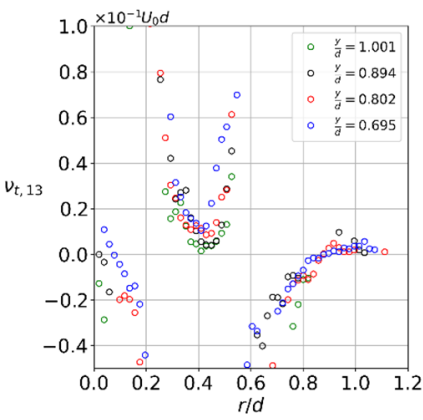

(f)

Figure 13. Turbulent viscosity coefficients for $(\mathbf{a}-\mathbf{c})$ total and $(\mathbf{d}-\mathbf{f})$ stochastic turbulent fluctuations for high-swirl jet.

The turbulent diffusivity coefficients are provided in Figure 14. For the total fluctuations of the velocity and concentration, the maximum at $r / d \approx 0.6$ was related to the transport by coherent flow motion. The stochastic fluctuations were related to more uniform distribution with values between 0.01 and 0.03 of $U_{0} d$ in the outer mixing layer. Therefore, the evaluated turbulent Schmidt number (see Figure 15) for the stochastic fluctuations as being in the range of 0.2 to 0.5 .

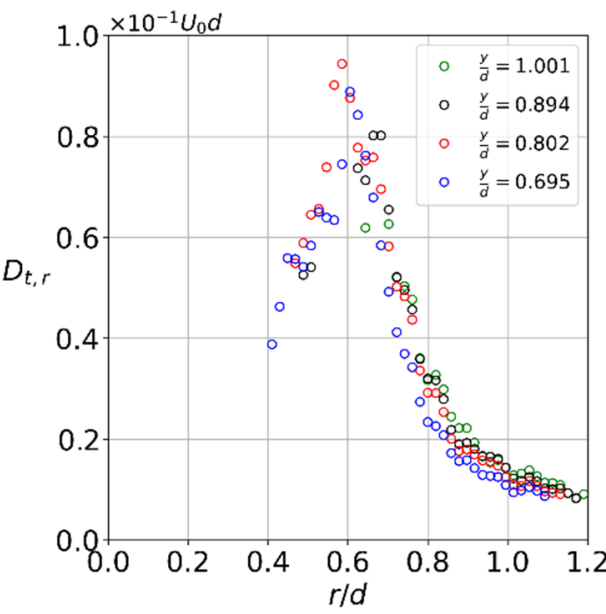

(a)

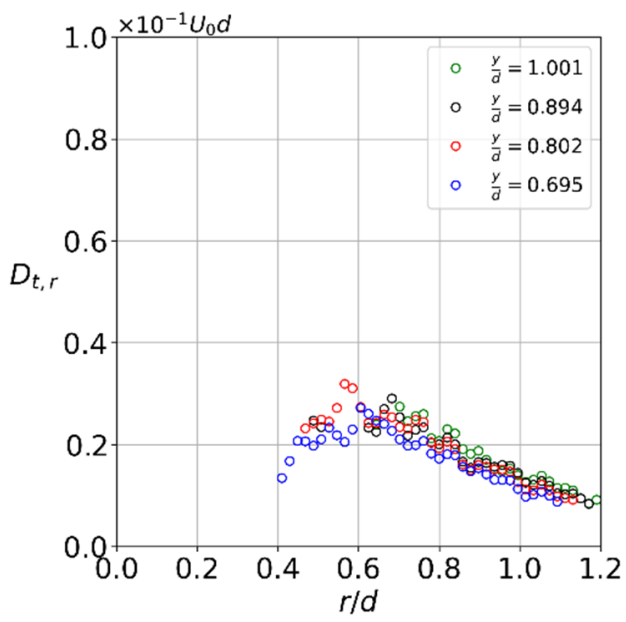

(b)

Figure 14. Turbulent diffusion coefficient for (a) total and (b) stochastic turbulent fluctuations for high-swirl jet. 


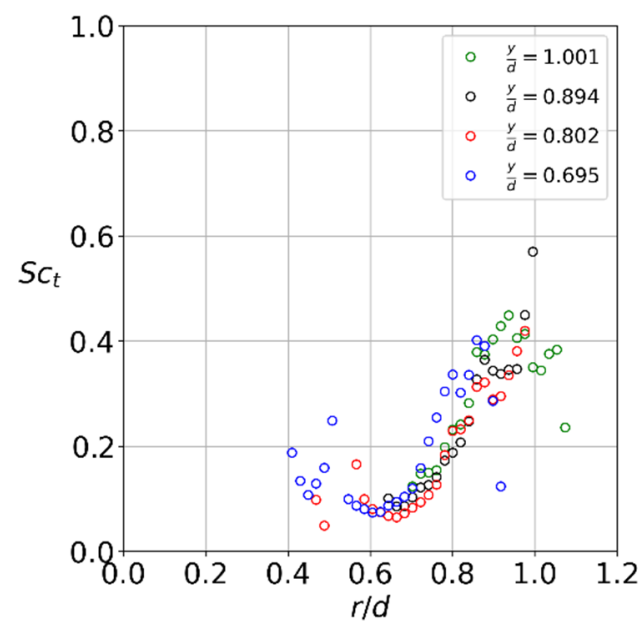

(a)

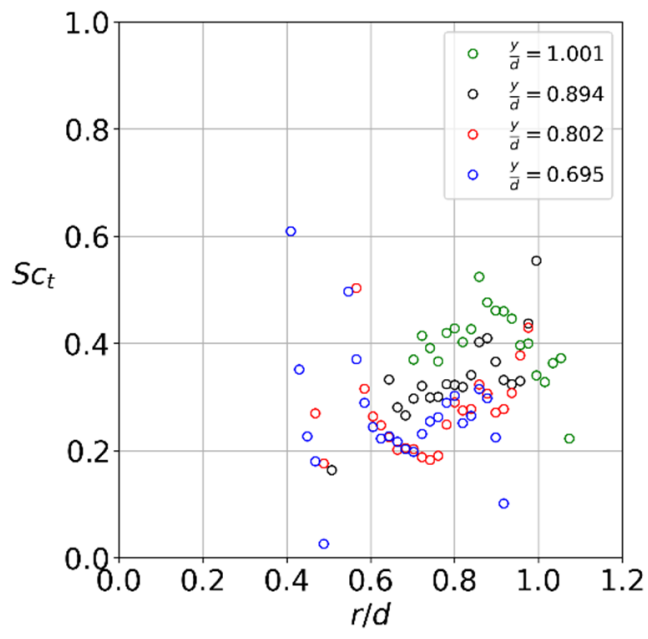

(b)

Figure 15. Turbulent Schmidt number for (a) total (b) stochastic turbulent fluctuations for high-swirl jet.

\subsection{Gas Turbine Swirler}

The shear Reynolds stresses for the gas turbine swirler are provided in Figure 16, where the distributions after the removal of the coherent flow fluctuations are also shown. Similar to the free swirling jet, the contribution of the coherent velocity fluctuations locally could exceed $80 \%$ for the momentum transport in the swirling main flow and in the outer mixing layer. For the shear layer between the nonswirling central jet and the rotating reverse flow, the contribution of the coherent fluctuations to the momentum turbulent transport $\left\langle u_{r}^{\prime} u_{y}^{\prime}\right\rangle$ and $\left\langle u_{y}^{\prime} u_{\theta}^{\prime}\right\rangle$ was much smaller (about $40 \%$ ). The radial mass flux of the central (methane) jet is shown in Figure 17. The contribution of the coherent structures was also about $40 \%$.

The evaluated turbulent viscosity and diffusivity coefficients are shown in Figures 18 and 19 for the methane central jet. Note that counter-gradient transport was also observed for the angular momentum. The effect of the coherent fluctuations was the strongest for the main annular swirling flow for the region $0.2<r / d<0.5$. Removal of the coherent fluctuations provided smaller scatter of the turbulent viscosity coefficients, which were typically below 0.05 of $U_{0} d$.

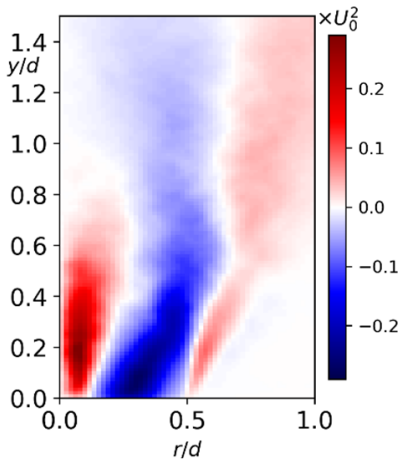

(a)

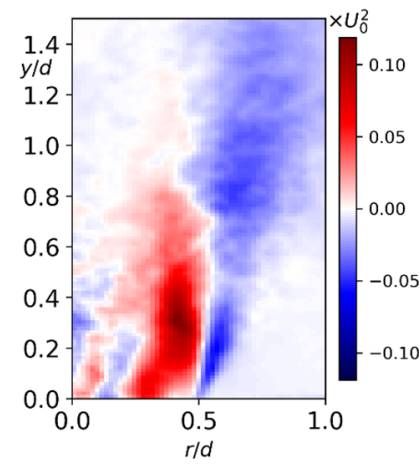

(b)

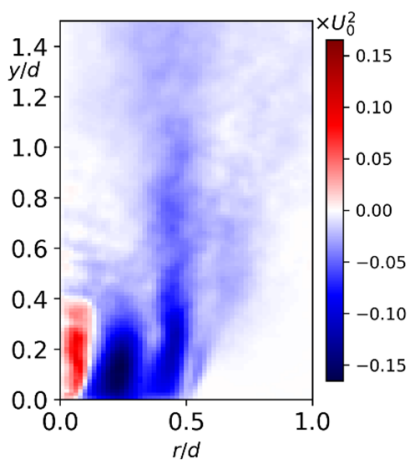

(c)

Figure 16. Cont. 


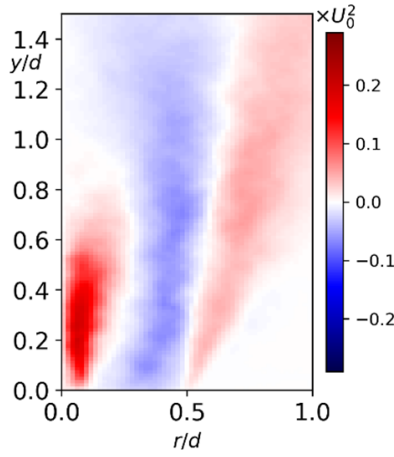

(d)

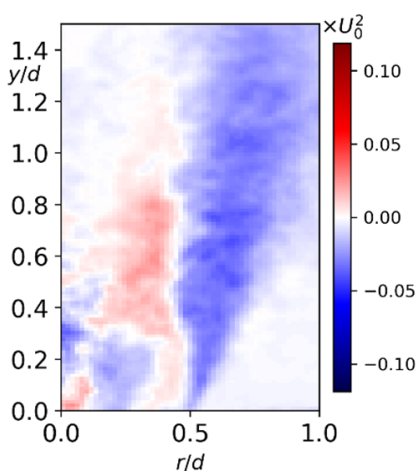

(e)

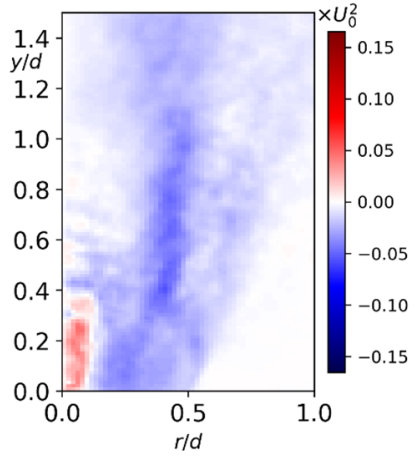

(f)

Figure 16. Distributions of Reynolds shear stresses (a) $\left\langle u_{r}^{\prime} u_{y}^{\prime}\right\rangle$, (b) $\left\langle u_{r}^{\prime} u_{\theta}^{\prime}\right\rangle$, (c) $\left\langle u_{y}^{\prime} u_{\theta}^{\prime}\right\rangle$, and the corresponding stochastic parts (d) $\left\langle u_{r}^{\prime \prime} u_{y}^{\prime \prime}\right\rangle$, (e) $\left\langle u_{r}^{\prime \prime} u_{\theta}^{\prime \prime}\right\rangle$ (f) $\left\langle u_{y}^{\prime \prime} u_{\theta}^{\prime \prime}\right\rangle$ for gas turbine swirler.

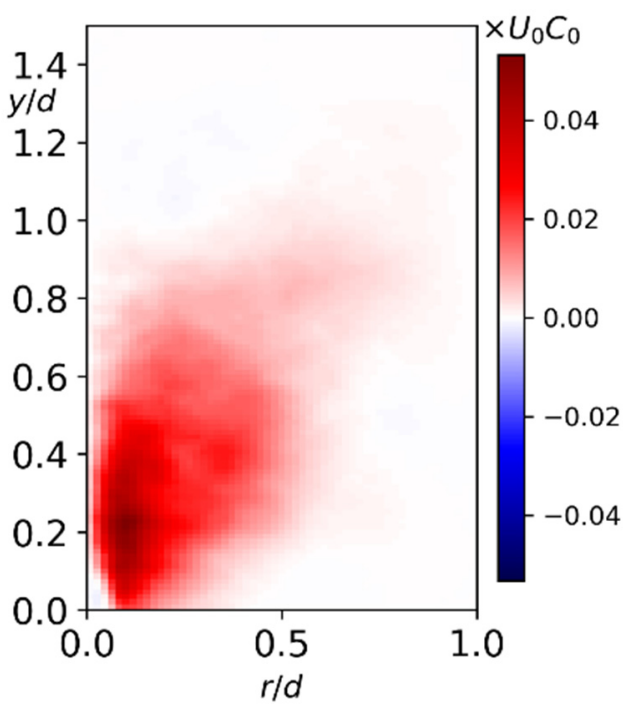

(a)

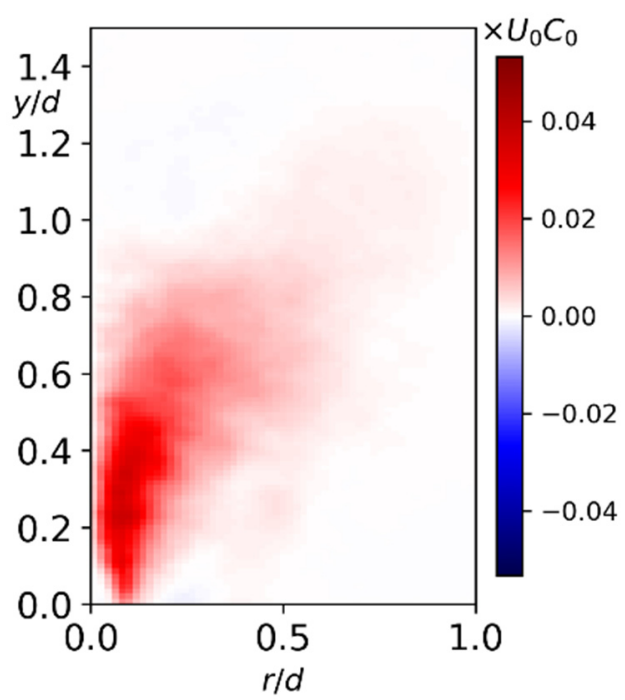

(b)

Figure 17. Radial turbulent flux of methane for (a) total and (b) stochastic turbulent fluctuations for gas turbine swirler.

For the central jet, the effect of the coherent fluctuations was not so strong for both the turbulent viscosity and turbulent diffusivity. For the stochastic fluctuations, the distribution appeared to be more uniform near the nozzle exit in comparison to the total fluctuations. The evaluated turbulent Schmidt number for the central jet of methane, neon, and air are shown in Figure 20 for both the total and stochastic fluctuations. Because the effect of the coherent fluctuations on the turbulent transport for the central jet was not strong, the turbulent Schmidt number values did not change significantly after removal of the coherent part. Comparing air and methane, the values were scattered around 0.6 and 0.8 , respectively, for the inner mixing layer center $r / d \approx 0.1$. 


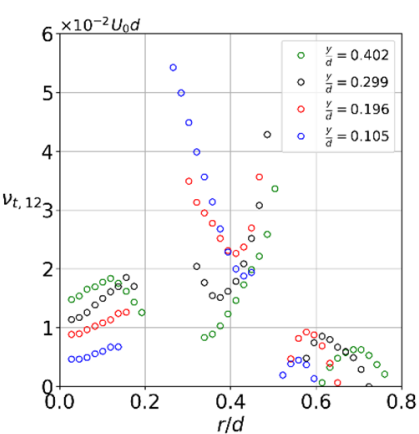

(a)

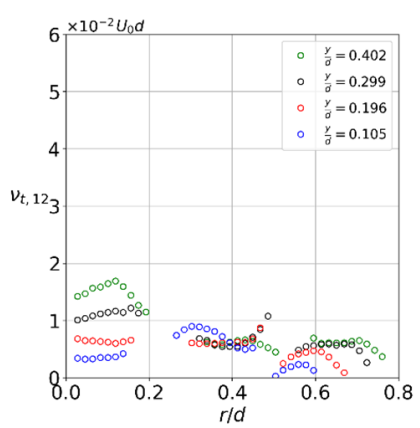

(d)

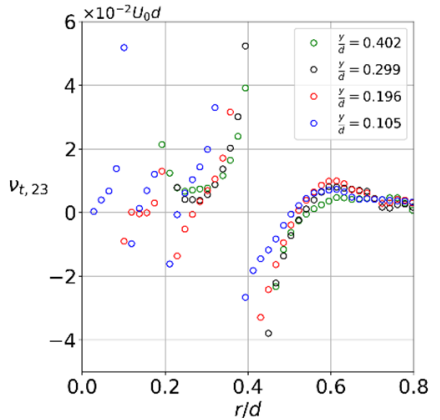

(b)

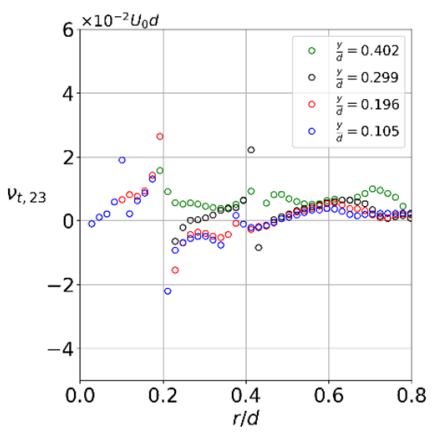

(e)

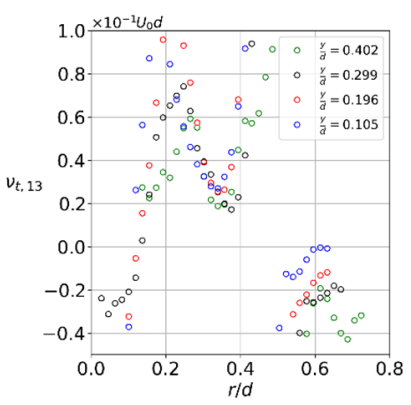

(c)

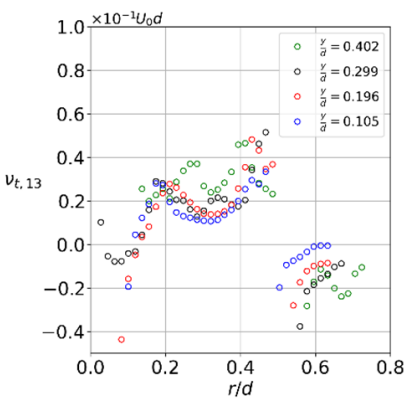

(f)

Figure 18. Turbulent viscosity coefficients for $(\mathbf{a}-\mathbf{c})$ total and $(\mathbf{d}-\mathbf{f})$ stochastic turbulent fluctuations for gas turbine swirler.

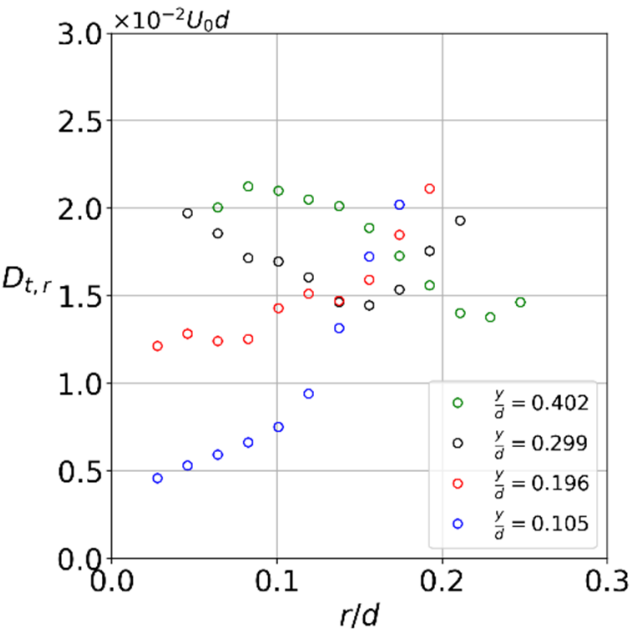

(a)

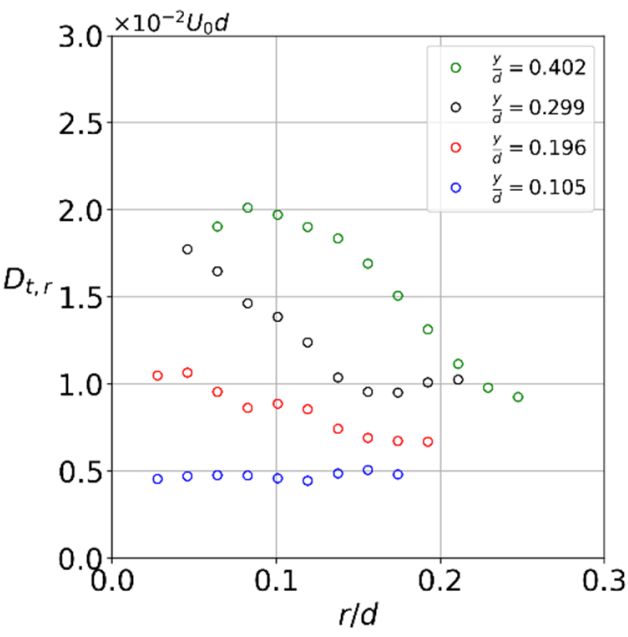

(b)

Figure 19. Turbulent diffusion coefficient for (a) total and (b) stochastic turbulent fluctuations for gas turbine swirler. 


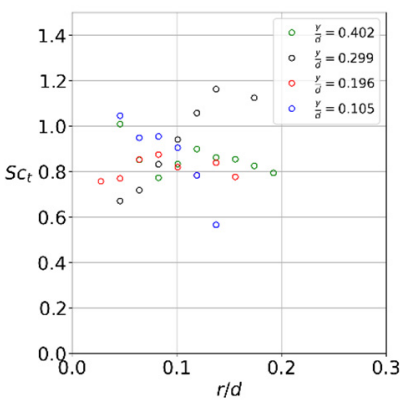

(a)

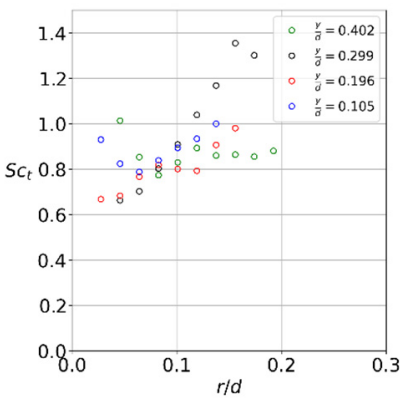

(d)

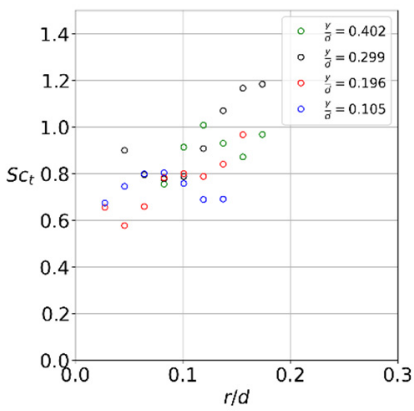

(b)

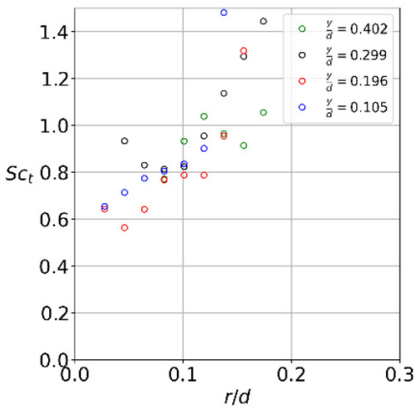

(e)

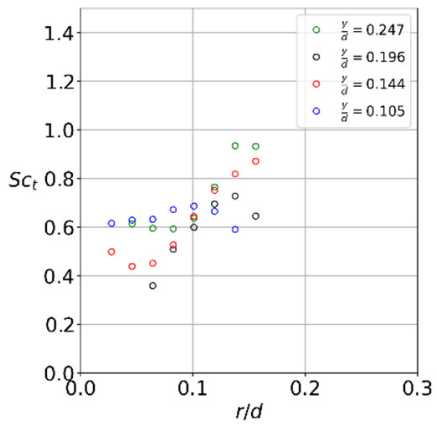

(c)

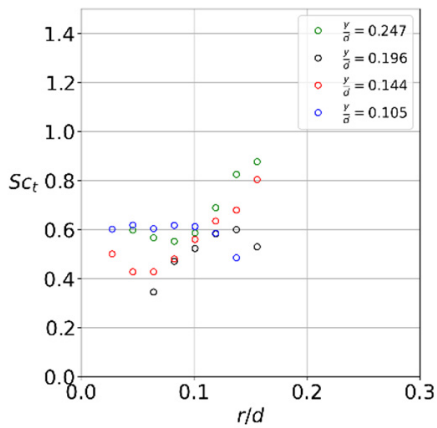

(f)

Figure 20. Turbulent Schmidt number for (a) methane, (b) neon, (c) air, and (d-f) the corresponding stochastic parts for gas turbine swirler.

\section{Discussion}

For the free air jet, it was observed that the swirl promoted the mass and momentum exchange between the jet and surrounding air. Therefore, the turbulent Schmidt number $S c_{t}$ distribution became more uniform with the scatter in the range of 0.2 to 0.8 . After the vortex breakdown, the mixing was even more enhanced due to the intensive pulsations associated with coherent flow structures. By accounting for the unsteady flow motion and removing the coherent flow pulsations, it was found that the $S c_{t}$ values were close to 0.4 for the stochastic fluctuations. For high-swirl jet flow, it was observed that counter-gradient transport took place for $\left\langle u_{y}^{\prime} u_{\theta}^{\prime}\right\rangle$, as has been observed previously, for example, for the rotating flow in a pipe [32].

The counter-gradient turbulent transport was also observed for the swirling flow of the gas turbine combustor. Similar to the high-swirl free jets, intensive coherent pulsations were found to contribute substantially to the local turbulent transport for the main annular swirling flow, whereas their effect for the mass and momentum transport of the central nonswirling pilot jet was not so strong. The turbulent Schmidt number was also evaluated for the different gases supplied from the central jet. It was found to be slightly higher for the methane jet (about 0.8) compared to the air jet (about 0.6).

\section{Conclusions}

The present study reports on the experimental investigation of turbulent transport in a free swirling jet and for a model gas turbine combustor using simultaneously PIV and PLIF measurements. Based on the instantaneous velocity and concentration snapshots, the Reynolds stresses and fluxes were evaluated. The data obtained was useful for the validation of numerical models. The turbulent viscosity and diffusivity coefficients were evaluated for basic gradient turbulent transport models (BH and GDH). The turbulent Schmidt number $S c_{t}$ was evaluated as the ratio of the turbulent viscosity and turbulent diffusivity. Based on the POD, the effect of coherent flow fluctuations on the turbulent 
viscosity and diffusivity coefficients was also evaluated. The high-swirl flows with vortex breakdown for the free jet configuration and for the combustion chamber were characterized by intensive turbulent fluctuations, which contributed substantially to the local turbulent transport of mass and momentum. Moreover, the high-swirl flows were found to be characterized by counter-gradient transport (i.e., negative turbulent viscosity) for $\left\langle u_{y}^{\prime} u_{\theta}^{\prime}\right\rangle$ near the jet axis and in the outer region of the mixing layer.

Author Contributions: A.S., A.L., D.S. and V.D. have equally contributed to the development of the present paper, including the conceptualization, methodology, data acquisition and analysis, interpretation of the results, and manuscript preparation. All authors have read and agreed to the published version of the manuscript.

Funding: This research was funded by the Russian Science Foundation, grant number 19-79-10225. The equipment was provided within the frame of the state contract with IT SB RAS.

Data Availability Statement: The data presented in this study are available on request from the corresponding author.

Acknowledgments: The authors thank Kemal Hanjalić for his important suggestions.

Conflicts of Interest: The authors declare no conflict of interest.

\section{References}

1. Lucca-Negro, O.; O’Doherty, T. Vortex breakdown: A review. Prog. Energy Combust. Sci. 2001, 27, 431-522. [CrossRef]

2. Syred, N. A review of oscillation mechanisms and the role of the precessing vortex core (PVC) in swirl combustion systems. Prog. Energy Combust. Sci. 2006, 32, 93-161. [CrossRef]

3. Dunn-Rankin, D. Lean Combustion: Technology and Control; Academic Press: Cambridge, UK, 2008.

4. Lieuwen, T.; Torres, H.; Johnson, C.; Zinn, B.T. A mechanism of combustion instability in lean premixed gas turbine combustors. J. Eng. Gas Turb. Power 2001, 123, 182-189. [CrossRef]

5. Meier, W.; Weigand, P.; Duan, X.R.; Giezendanner-Thoben, R. Detailed characterization of the dynamics of thermoacoustic pulsations in a lean premixed swirl flame. Combust. Flame 2007, 150, 2-26. [CrossRef]

6. Huang, Y.; Yang, V. Dynamics and stability of lean-premixed swirl-stabilized combustion. Prog. Energy Combust. Sci. 2009, 35, 293-364. [CrossRef]

7. Estefanos, W.; Bhayaraju, U.; Hamza, M.; Jeng, S.M. Evaluation of Two Measurement Techniques to Quantify Fuel-Air Mixing of a Gas Turbine Pre-mixer at Atmospheric Conditions. In Turbo Expo: Power for Land, Sea, and Air; American Society of Mechanical Engineers: New York, NY, USA, 2015; Volume 56680, p. V04AT04A060. [CrossRef]

8. Schulz, C.; Sick, V. Tracer-LIF diagnostics: Quantitative measurement of fuel concentration, temperature and fuel/air ratio in practical combustion systems. Prog. Energy Combust. Sci. 2005, 31, 75-121. [CrossRef]

9. Stohr, M.; Sadanandan, R.; Meier, W. Experimental study of unsteady fame structures of an oscillating swirl fame in a gas turbine model combustor. Proc. Combust. Inst. 2009, 32, 2925-2932. [CrossRef]

10. Renaud, A.; Yokomori, T.; Tachibana, S. Study of a thermo-acoustic instability triggering in a low-swirl burner using simultaneous time-resolved acetone and OH-PLIF. Proc. Combust. Inst. 2019, 37, 2627-2633. [CrossRef]

11. Felden, A.; Riber, E.; Cuenot, B. Impact of direct integration of Analytically Reduced Chemistry in LES of a sooting swirled non-premixed combustor. Combust. Flame 2018, 191, 270-286. [CrossRef]

12. Agostinelli, P.W.; Laera, D.; Boxx, I.; Gicquel, L.; Poinsot, T. Impact of wall heat transfer in Large Eddy Simulation of flame dynamics in a swirled combustion chamber. Combust. Flame 2021, 234, 111728. [CrossRef]

13. Hartmann, H.; Derksen, J.J.; Van den Akker, H.E.A. Mixing times in a turbulent stirred tank by means of LES. AIChE J. 2006, 52, 3696-3706. [CrossRef]

14. Gualtieri, C.; Angeloudis, A.; Bombardelli, F.; Jha, S.; Stoesser, T. On the values for the turbulent Schmidt number in environmental flows. Fluids 2017, 2, 17. [CrossRef]

15. Daly, B.J.; Harlow, F.H. Transport equations in turbulence. Phys. Fluids 1970, 13, 2634-2649. [CrossRef]

16. Jakirlić, S.; Hanjalić, K.; Tropea, C. Modeling rotating and swirling turbulent flows: A perpetual challenge. AIAA J. 2002, 40, 1984-1996. [CrossRef]

17. Hanjalić, K.; Launder, B.E. A Reynolds stress model of turbulence and its application to thin shear flows. J. Fluid Mech. 1972, 52, 609-638. [CrossRef]

18. Paglianti, A.; Montante, G. Simultaneous measurements of liquid velocity and tracer concentration in a continuous flow stirred tank. Chem. Eng. Sci. 2020, 216, 115495. [CrossRef]

19. Hitimana, E.; Fox, R.O.; Hill, J.C.; Olsen, M.G. Experimental characterization of turbulent mixing performance using simultaneous stereoscopic particle image velocimetry and planar laser-induced fluorescence. Exp. Fluids 2019, 60, 28. [CrossRef] 
20. Lobasov, A.S.; Alekseenko, S.V.; Markovich, D.M.; Dulin, V.M. Mass and momentum transport in the near field of swirling turbulent jets. Effect of swirl rate. Int. J. Heat Fluid Flow 2020, 83, 108539. [CrossRef]

21. Janus, B.; Dreizler, A.; Janicka, J. Experimental study on stabilization of lifted swirl flames in a model GT combustor. Flow Turbul. Combust. 2005, 75, 293-315. [CrossRef]

22. Sharaborin, D.K.; Savitskii, A.G.; Bakharev, G.Y.; Lobasov, A.S.; Chikishev, L.M.; Dulin, V.M. PIV/PLIF investigation of unsteady turbulent flow and mixing behind a model gas turbine combustor. Exp. Fluids 2021, 62, 96. [CrossRef]

23. Scarano, F. Iterative image deformation methods in PIV. Meas. Sci. Technol. 2001, 13, R1-R19. [CrossRef]

24. Soloff, S.M.; Adrian, R.J.; Liu, Z.C. Distortion compensation for generalized stereoscopic particle image velocimetry. Meas. Sci. Technol. 1997, 8, 1441-1454. [CrossRef]

25. Sirovich, L. Turbulence and the dynamics of coherent structures. I. Coherent structures. Q. Appl. Math. 1987, 45, 561-571. [CrossRef]

26. Kerschen, G.; Golinval, J.C.; Vakakis, A.F.; Bergman, L.A. The method of proper orthogonal decomposition for dynamical characterization and order reduction of mechanical systems: An overview. Nonlinear Dyn. 2005, 41, 147-169. [CrossRef]

27. Van Oudheusden, B.W.; Scarano, F.; Van Hinsberg, N.P.; Watt, D.W. Phase-resolved characterization of vortex shedding in the near wake of a square-section cylinder at incidence. Exp. Fluids 2005, 39, 86-98. [CrossRef]

28. Alekseenko, S.V.; Dulin, V.M.; Kozorezov, Y.S.; Markovich, D.M. Effect of high-amplitude forcing on turbulent combustion intensity and vortex core precession in a strongly swirling lifted propane/air flame. Combust. Sci. Technol. 2012, 184, 1862-1890. [CrossRef]

29. Hussain, A.K.M.F.; Reynolds, W.C. The mechanics of an organized wave in turbulent shear flow. J. Fluid Mech. 1970, 41, 241-258. [CrossRef]

30. Duwig, C.; Fuchs, L. Large eddy simulation of vortex breakdown/flame interaction. Phys. Fluids 2007, 19, 075103. [CrossRef]

31. Markovich, D.M.; Dulin, V.M.; Abdurakipov, S.S.; Kozinkin, L.A.; Tokarev, M.P.; Hanjalić, K. Helical modes in low-and high-swirl jets measured by tomographic PIV. J. Turbul. 2016, 17, 678-698. [CrossRef]

32. Kitoh, O. Experimental study of turbulent swirling flow in a straight pipe. J. Fluid Mech. 1991, 225, 445-479. [CrossRef] 